\title{
CREATION OF GOAL SCORING OPPORTUNITIES BY MEANS OF DIFFERENT TYPES OF OFFENSIVE ACTIONS IN US MAJOR LEAGUE SOCCER
}

original paper

doi: https://doi.org/10.5114/hm.2017.73616

\author{
JOAQUÍN GONZALEZ-RODENAS ${ }^{1}$, IGNACIO LOPEZ-BONDIA ${ }^{1}$, \\ FERRAN CALABUIG ${ }^{1}$, JOSE ANTONIO PÉREZ-TURPIN ${ }^{2}$, RAFAEL ARANDA ${ }^{1}$ \\ ${ }^{1}$ Department of Physical and Sports Education, University of Valencia, Valencia, Spain \\ ${ }^{2}$ Physical Education and Sports Department, Faculty of Education, University of Alicante, Alicante, Spain
}

\begin{abstract}
Purpose. The aim of the study was to describe how scoring opportunities emerge and finish in Major League Soccer, taking into account offensive and defensive tactical indicators.

Methods. The total of 360 team possessions that led to scoring opportunities during 30 random matches from Major League Soccer were analysed. For each possession, 14 dimensions were evaluated by means of observational methodology. Results. The scoring opportunities were created in $52.8 \%$ by organized attacks, in $25.3 \%$ by counterattacks, and in $21.9 \%$ by set pieces. Organized attacks were characterized by starting in non-invasive zones (57.1\%), performing a non-penetrative action (72.6\%), and making 4 or more passes (70.1\%), while counterattacks started primarily in invasive zones (87.9\%) and were bound with performing a penetrative action (96.7\%) and making 3 or less passes (70.3\%). Chi-square analysis showed that scoring opportunities created by organized attacks had more proportion of previous actions in wide areas $(p=0.034)$, while counterattacks showed more passes in behind the defence from central areas $(p=0.001)$. As for the final action, counterattacks achieved more penetration over the opponent defence than organized attacks $(p=0.036)$ but for both types of attacks, the opponent defensive zone was the space majorly used to finish. For set pieces, corner kicks created $46.8 \%$ and free kicks $45.6 \%$ of the scoring opportunities.

Conclusions. Goal scoring opportunities from organized attacks started more frequently in non-invasive zones, showed a greater proportion of non-penetrative actions, were built up by means of longer passing sequences, used more the wide areas of the pitch, and achieved less penetration over the opponent than those from counterattacks.
\end{abstract}

Key words: football, match analysis, game performance analysis

\section{INTRODUCTION}

The primary objective of performance analysis is to support coaches and players in the decision-making process by providing relevant information regarding performance [1]. Soccer is an invasion sport where breaking the opponent's lines to progress and finish the attack in order to score as many goals as possible is the main offensive objective. Therefore, a large number of studies have analysed tactical indicators related to goal scoring in soccer [2-4]. However, although goal scoring is the main indicator of success in soccer, it may not truly represent the underlying tactical strat- egies of a team, i.e. those that are concerned with the actual development of goal scoring opportunities [5]. Tenga et al. [6] showed how scoring opportunities could be used as a proxy for goals scored when comparing the effectiveness of different playing tactics in soccer.

Few studies have specifically described offensive sequences that lead to shots at goal or scoring opportunities in elite soccer. Previous research found that the majority of shots at goal were created in short passing sequences [7], started in the opponent half [8], and used more frequently the positional attack $[9,10]$. However, although the opposing team has a key in-

Correspondence address: Rafael Aranda, Department of Physical and Sports Education, Faculty of Physical Activity and Sport Sciences, University of Valencia, Gascó Oliag 3, 46010 Valencia, Spain, e-mail: Rafael.Aranda@uv.es

Received: November 29, 2017

Accepted for publication: January 2, 2018

Citation: Gonzalez-Rodenas J, Lopez-Bondia I, Calabuig F, Pérez-Turpin JA, Aranda R. Creation of goal scoring opportunities by means of different types of offensive actions in us major league soccer. Hum Mov. 2017;18(5)special/issue:106-116 doi: https://doi.org/10.5114/hm.2017.73616. 
fluence on the offensive behaviour, very few studies have included the analysis of performance indicators related to the opponent position and behaviour [11, 12]. In this line, Olsen and Larsen [13] showed that more scoring opportunities and goals were created from breakdown attacks (counterattacks) started when the opposition defence was imbalanced rather than balanced. Tenga et al. [14] observed that counterattacks were more effective than elaborate attacks only when playing against an imbalanced defence, and not against a balanced defence in the Norwegian soccer league. Also, González-Ródenas et al. [10] observed that scoring opportunities produced by counterattacks took place in more invasive zones than those produced by organized attacks. González-Ródenas et al. [11] performed an interesting multifactorial study focused on counterattacks in which initial pressure was one of the tactics that influenced performance. More research is necessary to understand the key tactical indicators that lead to offensive success in soccer taking into account the opponent interaction.

Furthermore, it is interesting to examine the performance indicators of different competitions in order to help coaches to enhance the training process as well as the competitive strategies. In this aspect, United States Major League Soccer (MLS) has been considered of special interest because it is an emergent soccer league [15]. However, this competition has not been widely explored scientifically with regard to its tactical indicators and creation of offensive success.

The aim of this study was to describe how scoring opportunities emerge and finish in MLS, taking into account not only offensive tactical indicators but also some defensive ones of the opponent team.

\section{MATERIAL AND METHODS}

\section{Sample}

MLS is a club domestic league in which 20 teams participated during the 2014 season. Each match from the whole (first and second round) regular MLS 2014 was assigned with a number from 1 to 360 . An online random number generator [16] was used to select 30 matches; for the first 15 , the home team was analysed and for the second 15, the away team was evaluated. The selected matches were downloaded from the Wyscout platform (Wyscout Spa, Italy). From these 30 matches, 360 'team possessions' that led to the creation of goal scoring opportunities were analysed with the consideration of the definition of Pollard and Reep [17].

It was considered that a scoring opportunity was

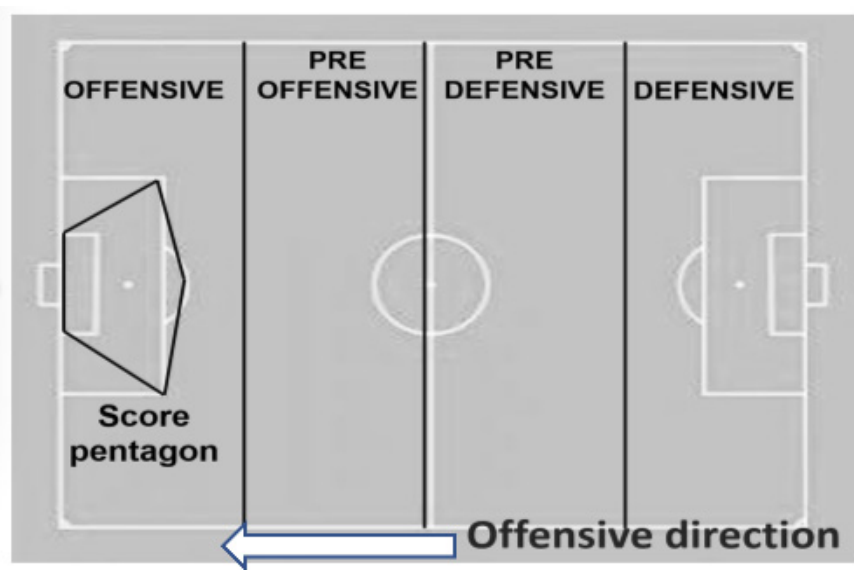

Figure 1. Zones of the field and score pentagon

created when the team had a chance of scoring a goal during the team possession. This includes all shots produced inside the score pentagon (Figure 1) and those produced outside the score pentagon that passed near the goal (2 meters or less with respect to the goal). The score pentagon is defined as the zone within the official soccer field that selects the space with high shooting angle and short distance to goal (20 meters or less), which are very important factors to achieve a goal $[17,19]$. All chances of shooting inside the score pentagon are considered scoring opportunities if the player is facing the goal, there are not any opponents between him and the goal, as well as the player has enough space and time to make a playing decision.

\section{Variables}

Firstly, three contextual variables were considered: location (home, away), time (first half, second half) and score (losing, drawing, wining). Team possessions were analysed according to the type of attack (organized attack, counterattack, and set piece) (Table 1). Secondly, 15 tactical indicators were analysed to describe the 'possession start,' 'opponent defensive situation,' 'possession development,' and 'finishing' of counterattacks and organized attacks (Table 2). Regarding the analysis of set pieces, 2 variables were analysed (type of start and goal effectiveness).

\section{Match performance analysis}

For the analysis, a soccer coach or researcher experienced in match performance analysed each possession post-event as many times as necessary using selected variables from the REOFUT observational instrument [23]. In addition, Lince software [24] was applied to register and save data. The reliability of data 


\section{HUMAN MOVEMENT}

J. Gonzalez-Rodenas et al., Scoring attempts in US Major League Soccer

Table 1. Categories and descriptions for the 'type of attack' dimension

Type of attack: Type of offensive action depending on the state of the ball (in play or open play vs. out of play or restart) at the start of the attack and the degree of offensive directness $[14,18]$ in the offensive process. Three categories were considered:

a. Organized attack: (1) The possession starts by winning the ball in play or restarting the game; (2) this kind of possession allows the opponent to have more opportunity to minimize surprise, reorganize their system, and be prepared defensively; (3) the progression towards the opponent's goal can be combinative (high percentage of non-penetrative and short passes and long duration) or direct (long pass, evaluated qualitatively).

b. Counterattack: (1) The possession starts by winning the ball in play; (2) the first or second player in action tries to penetrate using penetrative passes or dribbles; (3) the progression towards the opponent's goal has high percentage of penetrative passes and short duration (evaluated qualitatively); (4) this kind of possession tries not to allow the opponent to have opportunity to minimize surprise, reorganize their system, and be prepared defensively.

c. Set-plays: (1) There is a restart in the opponent's half; (2) the tactical situation of the attacking team is prepared to try to shot at goal (both teams group players into or just in front of the box and player positions change because some of the defenders move forward to try to shot at goal); (3) the attacking team tries to cross the ball towards the box or shot at goal in one or two passes. (All corner kicks, all penalty kicks, and free kicks with the above characteristics are considered in this category.)

was calculated with the intra- and inter-observer agreement (Cohen's kappa) by analysing 54 random possessions from the study. These were assessed by the same observer after a gap of time longer than 1 month to obtain intra-observer reliability. Inter-observer reliability was achieved by comparing data from the same 54 possessions, but observed by another experienced observer from our research group. The data showed high levels of reliability in accordance with Altman's criteria [25] (inter-observer reliability: 0.7710.973; intra-observer reliability: 0.768-1.00). The conducted research is not related to either human or animals use.

\section{Statistical analysis}

Data collected on Lince software were transcribed to a database created in the SPSS 20.0 program (SPSS, Chicago, USA). Firstly, a frequency analysis was carried out to describe the dimensions and categories related to 'possession start,' 'opponent defensive situation,' 'possession development,' and 'set pieces.' Secondly, a chi-square analysis was performed to observe the differences between counterattacks and organized attacks for the dimensions related to 'finishing.' Data were presented as frequencies and percentages of each category.

\section{Ethical approval}

The research related to human use has been complied with all the relevant national regulations, institutional policies and in accordance the tenets of the Helsinki Declaration, and has been approved by the

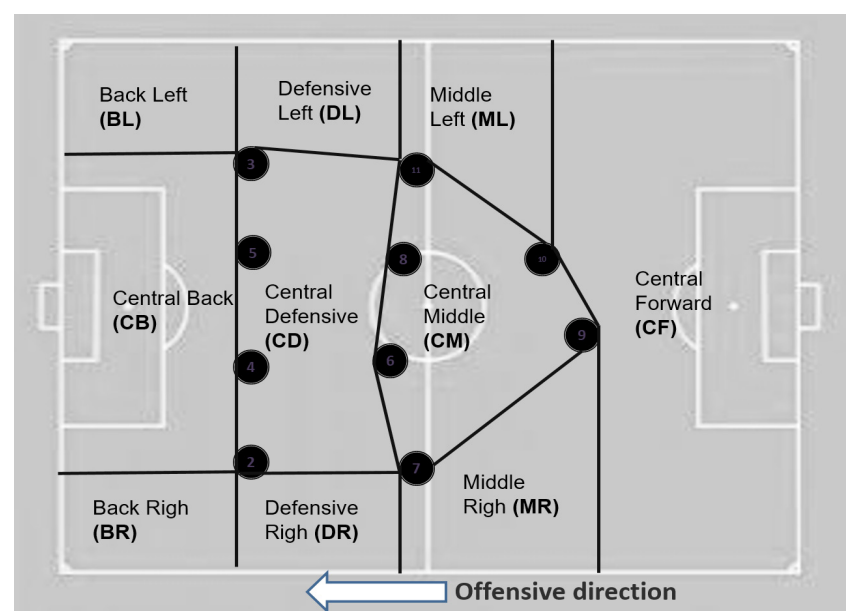

Figure 2. Space of defensive occupation that define the level of invasion over the opponent. These zones are dynamic and change every second depending on the positioning of the opposing players

authors' institutional review board or equivalent committee.

\section{RESULTS}

Table 3 shows the contextual characteristics of the scoring opportunities analysed in the study. A bigger proportion of scoring opportunities were created by means of organized attacks (64.9\%) when the teams were losing in comparison with drawing (47.5\%) and winning (42.0\%). Related to this, the proportion of counterattacks was very low when the teams were losing (14.5\%) and much higher when the teams were winning (42\%). Additionally, teams created less oppor- 
Table 2. Categories and descriptions for the dimensions of 'possession start,' 'opponent defensive situation,' 'possession development,' and 'finishing'

\section{Possession start}

1. Type of start: Seven categories were considered: (a) turnover won: when the defender collects, somewhere in the pitch, a ball lost (clearances or missed passes) by the opposing team [20]; (b) stealing the ball: (1) when the defender prevents a ball passed by an opponent from reaching its intended receiver by contacting the ball and keeping his own team in possession of the ball [21] or (2) when the defender dispossesses the opponent of the ball through a physical challenge or defensive pressure [21]; (c) goal kick; (d) throw in; (e) free kick; (f) kick off; (g) penalty kick.

2. Field starting zone: Area of the playing field where team possession starts (Figure 1). Four areas were considered [22, 23]: (a) defensive; (b) pre-defensive; (c) pre-offensive; (d) offensive (score pentagon included).

3. Initial penetration: Degree of offensive directness in the first 3 seconds of the team possession [23]: (a) penetrative action: passes or dribbles towards the opponent's goal past opponent player(s) performed during the first 3 seconds of the ball possession;

(b) non-penetrative action: any technical action towards any direction that does not overcome any opponent player(s) during the first 3 seconds of the ball possession.

\section{Opponent defensive situation}

4. Initial opponent position: Opponent's height (longitudinal) position on the field when the team possession starts (excluding goalkeeper): (a) advanced: the opponent has the most backward player in the opposing half (closer to their opponent goal than to the own goal); (b) medium: the opponent has the most backward player closer to the midline than to their own goal; (c) back: the opponent has the most backward player closer to their own goal line than the midline.

5. Initial invasive zone: Area within the space of defensive occupation of the opponent [22] where team possession starts (Figure 2): (a) non-invasive zone: the possession starts in the CF zone; (b) invasive zone: the possession starts in the CM, ML, or MR zone; (c) very invasive zone: the possession starts in the $\mathrm{CD}, \mathrm{DL}, \mathrm{DR}, \mathrm{CB}, \mathrm{BL}$, or BR zone.

6. Initial opponent number: Number of defending players located between the ball and their goal when possession starts (excluding goalkeeper): (a) micro-group (3 or less defending players); (b) meso-group (4-6 defending players); (c) macro-group (7 or more defending players).

7. Initial opponent pressure: Distance between the player with the ball (first attackers) and an immediate pressing opponent player(s) (first defender(s)) during the first 3 seconds of the ball possession: (a) pressure: 1 or several opponent players pressure the attackers within the first 3 seconds of the possession (the defender(s) are always located within

1.5 meters of the first attackers; 1.5 meters has been estimated close enough to hamper a pass forward and close enough to steal the ball); (b) no pressure: none of the players pressures the attackers during the first 3 seconds of the possession.

\section{Possession development}

8. Passes per possession: Passes performed by players during team possession: (a) short possession ( 3 or less passes); (b) medium possession (4-6 passes); (c) long possession (7 or more passes).

9. Percentage of penetrative passes: Percentage of passes past the opponent player(s) in relation to the total number of passes during team possession: (a) low penetrating possession (0-33\%); (b) medium penetrating possession (34-66\%); (c) high penetrating possession (67-100\%).

\section{Finishing}

10. Penultimate invasive zone (penetration level): Area within the space of defensive occupation of the opponent [21] where the penultimate player of the possession performs his action (Figure 2): (a) back zone (CB); (b) defensive (DL, CD, DR); (c) middle defensive (ML, CM, MR); (d) forward (CF).

11. Penultimate invasive zone (width level): Area within the space of defensive occupation of the opponent [21] where the penultimate player of the possession performs his action (Figure 2): (a) wide areas (DR, MR, DL, ML); (b) central areas (CB, CD, CM, CF).

12. Penultimate action: Tactical action performed by the player who passed the ball to the player who performed the last action: (a) normal pass: pass towards any directions that does not overcome any opponent player(s); (b) penetrative pass: pass towards the opponent's goal past opponent player(s); (c) pass in behind the defence: pass from inside zones of the space of defensive occupation (central defensive and central middle) that tries to penetrate towards the central back zone (Figure 2); (d) cross: pass from outside zones (not central defensive or central middle) of the space of defensive occupation (Figure 2) towards the score pentagon (Figure 1); (e) other: other action different from the above ones.

13. Last invasive zone (penetration level): Area within the space of defensive occupation of the opponent [21] where the last player of the possession performs his action (Figure 2): (a) back zone (CB); (b) defensive (DL, CD, DR); (c) middle defensive (ML, CM, MR); (d) forward (CF).

14. Goal effectiveness: Describes if the scoring opportunities achieved goal. Two categories: (a) goal; (b) no goal.

15. Last invasive zone effectiveness: Describes the percentage of scoring opportunities that achieved goal in each specific area within the space of defensive occupation (Figure 2). 


\section{HUMAN MOVEMENT}

J. Gonzalez-Rodenas et al., Scoring attempts in US Major League Soccer

Table 3. Description and categories for the dimensions related to the contextual variables

\begin{tabular}{|c|c|c|c|c|c|c|c|c|}
\hline \multirow{2}{*}{ Dimension } & \multirow{2}{*}{$n$} & \multicolumn{3}{|c|}{ Type of possession leading to score opportunities (\%) } & \multirow[t]{2}{*}{ Cramer's V } & \multirow{2}{*}{$d f$} & \multirow{2}{*}{$\chi^{2}$} & \multirow{2}{*}{$p$} \\
\hline & & Organized & Counterattack & Set pieces & & & & \\
\hline Location & & & & & 0.022 & 2 & 0.181 & 0.914 \\
\hline Home & 208 & 53.4 & 25.5 & 21.2 & & & & \\
\hline Away & 152 & 52.0 & 25.0 & 23.0 & & & & \\
\hline Time & & & & & 0.099 & 2 & 3.550 & 0.169 \\
\hline First half & 161 & 56.5 & 20.5 & 23.0 & & & & \\
\hline Second half & 199 & 49.7 & 29.1 & 21.1 & & & & \\
\hline Score & & & & & 0.248 & 4 & 22.057 & 0.001 \\
\hline Winning & 69 & 42.0 & 42.0 & 15.9 & & & & \\
\hline Drawing & 160 & 47.5 & 26.9 & 25.6 & & & & \\
\hline Losing & 131 & 64.9 & 14.5 & 20.6 & & & & \\
\hline
\end{tabular}

Table 4. Frequencies and percentages of the type of start and the type of attack

\begin{tabular}{lcccc} 
State of ball at start & $n(\%)$ & Type of attack & $n$ & Total \% \\
\multirow{2}{*}{ Open play } & & Counterattack & 91 & 25.3 \\
\cline { 3 - 5 } & $189(52.5)$ & Organized attack & 98 & 52.8 \\
\hline \multirow{2}{*}{ Restart } & $171(47.5)$ & Set pieces & 79 & 21.9 \\
\hline
\end{tabular}

tunities by means of set pieces while winning $(15.9 \%)$ than while drawing $(25.6 \%)$ or losing $(20.6 \%)$. No differences were found between the types of attack regarding 'game location' or 'time of the match.'

As presented in Table 4, organized attacks created $52.8 \%$ of the scoring opportunities, while counterattacks and set pieces created $25.3 \%$ and $21.9 \%$, respectively.

For organized attacks, the possession start was characterized by high frequency of turnovers won (43.7\%) and throws in (25.8\%), non-initial penetration in the first action (72.6\%), opponent in back position (43.2\%), a macro-group of opposing players between the ball and the goal (94.1\%), no pressure on the player with the ball (67.7\%), and starting in non-invasive zones (57.1\%). As for the possession development, a higher proportion of long possessions was found (42.6\%) (Table 5).

For counterattacks, stealing the ball from the opponent was the most common way to start the possession (68.1\%), while a high proportion of the initial actions took place in the pre-offensive (40.7\%) and pre-defensive $(36.3 \%)$ zones. The first actions were penetrative in $96.7 \%$ and occurred in invasion zones of the opponent in $74.7 \%$. Opposing teams were in advanced (47.3\%) and medium (41.8\%) positions, having a meso-group of players (4-7) between the ball and the goal (57.1\%) and not putting pressure on the player with the ball (83.5\%) (Table 5).

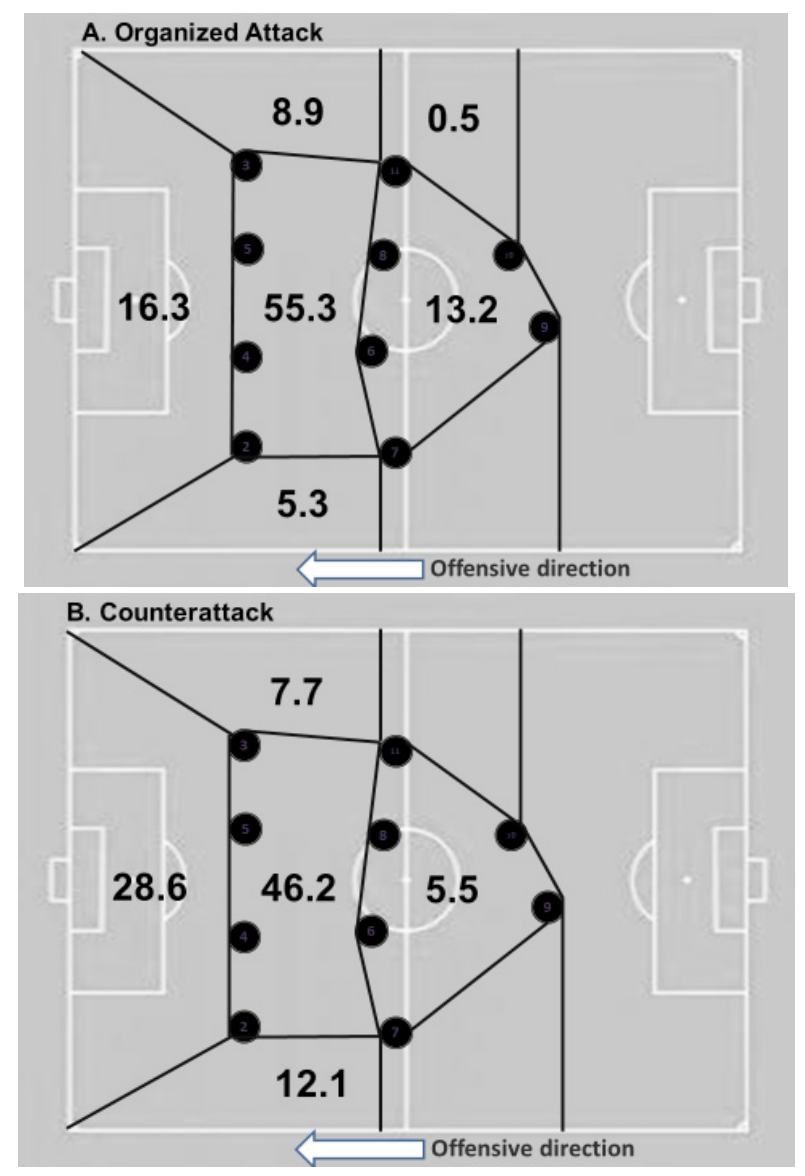

Figure 3. Percentage of scoring opportunities that took place for each invasive zone 
Table 5. Frequencies and percentages for the dimensions and categories of the study

\begin{tabular}{|c|c|c|c|c|c|c|c|}
\hline \multirow{2}{*}{ Dimension } & \multirow{2}{*}{ Category } & \multicolumn{2}{|c|}{ Total } & \multicolumn{2}{|c|}{ Organized attack } & \multicolumn{2}{|c|}{ Counterattack } \\
\hline & & $n$ & $\%$ & $n$ & $\%$ & $n$ & $\%$ \\
\hline \multicolumn{8}{|l|}{ Possession start } \\
\hline \multirow[t]{6}{*}{ Type of start } & Stealing the ball & 77 & 27.4 & 15 & 7.9 & 62 & 68.1 \\
\hline & Turnover won & 112 & 39.9 & 83 & 43.7 & 29 & 31.9 \\
\hline & Goal kick & 21 & 7.5 & 21 & 11.1 & & \\
\hline & Throw in & 49 & 17.4 & 49 & 25.8 & & \\
\hline & Free kick & 20 & 7.1 & 20 & 10.5 & & \\
\hline & Kick off & 2 & 0.7 & 2 & 1.1 & & \\
\hline \multirow[t]{4}{*}{ Field starting zone } & Defensive & 76 & 27.0 & 59 & 31.1 & 17 & 18.7 \\
\hline & Pre-defensive & 76 & 27.0 & 43 & 22.6 & 33 & 36.3 \\
\hline & Pre-offensive & 106 & 37.7 & 69 & 36.3 & 37 & 40.7 \\
\hline & Offensive & 23 & 8.2 & 19 & 10.0 & 4 & 4.4 \\
\hline \multirow[t]{2}{*}{ Initial penetration } & Penetrative action & 140 & 49.8 & 52 & 27.4 & 88 & 96.7 \\
\hline & Non-penetrative action & 141 & 50.2 & 138 & 72.6 & 3 & 3.3 \\
\hline
\end{tabular}

Opponent defensive situation

\begin{tabular}{|c|c|c|c|c|c|c|c|}
\hline \multirow{3}{*}{$\begin{array}{l}\text { Initial opponent } \\
\text { position }\end{array}$} & Advanced position & 84 & 34.1 & 41 & 26.5 & 43 & 47.3 \\
\hline & Medium position & 85 & 34.6 & 47 & 30.3 & 38 & 41.8 \\
\hline & Back position & 77 & 31.3 & 67 & 43.2 & 10 & 11.0 \\
\hline \multirow[t]{3}{*}{ Initial invasive zone } & Non-invasive zone & 103 & 40.9 & 92 & 57.1 & 11 & 12.1 \\
\hline & Invasive zone & 133 & 52.8 & 65 & 40.4 & 68 & 74.7 \\
\hline & Very invasive zone & 16 & 6.3 & 4 & 2.5 & 12 & 13.2 \\
\hline \multicolumn{2}{|c|}{ Initial opponent number Micro-group } & 5 & 2.0 & 0 & 0 & 5 & 5.5 \\
\hline & Meso-group & 61 & 24.9 & 9 & 5.9 & 52 & 57.1 \\
\hline & Macro-group & 180 & 73.1 & 146 & 94.1 & 34 & 37.4 \\
\hline \multirow[t]{2}{*}{ Initial pressure } & Pressure & 65 & 26.4 & 50 & 32.3 & 15 & 16.5 \\
\hline & No pressure & 181 & 73.6 & 105 & 67.7 & 76 & 83.5 \\
\hline \multicolumn{8}{|l|}{ Possession development } \\
\hline \multirow[t]{3}{*}{ Pass number } & Short possession & 119 & 42.3 & 55 & 28.9 & 64 & 70.3 \\
\hline & Medium possession & 78 & 27.8 & 54 & 28.4 & 24 & 26.4 \\
\hline & Long possession & 84 & 29.9 & 81 & 42.6 & 3 & 3.3 \\
\hline \multirow{3}{*}{$\begin{array}{l}\text { Percentage } \\
\text { of penetrating } \\
\text { passes }\end{array}$} & Low $(0-33 \%)$ & 87 & 31.0 & 79 & 41.6 & 8 & 8.8 \\
\hline & Medium (34-66\%) & 116 & 41.3 & 91 & 47.9 & 25 & 27.5 \\
\hline & High (67-100\%) & 78 & 27.8 & 20 & 10.5 & 58 & 63.7 \\
\hline
\end{tabular}

Regarding the moment of finishing the attacks, Table 6 shows that for organized attacks, in comparison with counterattacks, the previous player actions were carried out more frequently in wide areas $(50.5 \%$ vs. $37.9 \%)$, with higher proportion of crosses $(32.1 \%$ vs. $19.5 \%)$ and less proportion of passes in behind the defence (12.8\% vs. $28.7 \%)$. As for the last action, counterattacks achieved higher penetration over the opponent invasive zones so $28.6 \%$ of the scoring opportunities took place in the back zone of the opponent, while during the organized attacks this percentage equalled $16.4 \%$. However, no significant differences in goal ef- fectiveness were found although counterattacks tend to reach a higher proportion than organized attacks (13.2\% vs. $9.5 \%)$.

In a more detailed, descriptive, and visual analysis of the final action, Figure 3 shows how the defensive zone (space between the middle line and back line of the opponent) was the most used zone to finish the scoring opportunities for both organized attacks and counterattacks ( $55.3 \%$ and $46.2 \%$, respectively).

Regarding the goal effectiveness, the central back zone of the opponent achieved higher effectiveness in scoring goals for both counterattacks and organized 


\section{HUMAN MOVEMENT}

J. Gonzalez-Rodenas et al., Scoring attempts in US Major League Soccer

Table 6. Differences between organized attack and counterattack during the final action in scoring opportunities

\begin{tabular}{|c|c|c|c|c|c|c|c|c|}
\hline \multirow{2}{*}{ Dimension } & \multirow{2}{*}{$n$} & \multirow[t]{2}{*}{$\%$} & \multicolumn{2}{|c|}{ Type of attack (\%) } & \multirow{2}{*}{$\begin{array}{l}\text { Cramer's } \\
\text { V }\end{array}$} & \multirow{2}{*}{$d f$} & \multirow{2}{*}{$\chi^{2}$} & \multirow{2}{*}{$p$} \\
\hline & & & Organized & Counterattack & & & & \\
\hline $\begin{array}{l}\text { Penultimate invasive zone } \\
\text { (penetration level) }\end{array}$ & & & & & 0.073 & 1 & 1.478 & 0.478 \\
\hline Back zone (CB) & 0 & 0 & 0 & 0 & & & & \\
\hline Defensive (DL, CD, DR) & 165 & 60.0 & 62.2 & 55.2 & & & & \\
\hline Middle defensive (ML, CM, MR) & 98 & 35.6 & 34.0 & 39.1 & & & & \\
\hline Forward (CF) & 12 & 4.4 & 3.7 & 5.7 & & & & \\
\hline $\begin{array}{l}\text { Penultimate invasive zone } \\
\text { (width level) }\end{array}$ & & & & & 0.117 & 1 & 3.796 & 0.034 \\
\hline Wide areas (DR, MR, DL, ML) & 128 & (46.5) & 50.5 & 37.9 & & & & \\
\hline Central areas $(\mathrm{CB}, \mathrm{CD}, \mathrm{CM}, \mathrm{CF})$ & 147 & (53.5) & 49.5 & 62.1 & & & & \\
\hline Penultimate action & & & & & & 4 & 18.654 & 0.001 \\
\hline Normal pass & 24 & $(8.8)$ & 10.7 & 4.6 & & & & \\
\hline Penetrative pass & 111 & (40.5) & 38.0 & 46.0 & & & & \\
\hline Pass in behind the defence & 49 & (17.9) & 12.8 & 28.7 & & & & \\
\hline Cross & 77 & (28.1) & 32.1 & 19.5 & & & & \\
\hline Other & 14 & $(4.7)$ & 6.4 & 1.1 & & & & \\
\hline $\begin{array}{l}\text { Last invasive zone } \\
\text { (penetration level) }\end{array}$ & & & & & 0.174 & 3 & 8.515 & 0.036 \\
\hline Back zone (CB) & 57 & (20.4) & 16.4 & 28.6 & & & & \\
\hline Defensive (DL, CD, DR) & 192 & (69.8) & 69.8 & 65.9 & & & & \\
\hline Middle defensive (ML, CM, MR) & 31 & (13.5) & 13.2 & 5.5 & & & & \\
\hline Goal effectiveness & & & & & 0.056 & 1 & 0.890 & 0.228 \\
\hline Goal & 30 & 10.7 & 9.5 & 13.2 & & & & \\
\hline No goal & 251 & 89.3 & 90.5 & 86.8 & & & & \\
\hline
\end{tabular}

CB - central back, DL - defensive left, CD - central defensive, DR - defensive right, ML - middle left,

$\mathrm{CM}$ - central middle, MR - middle right, $\mathrm{CF}$ - central forward

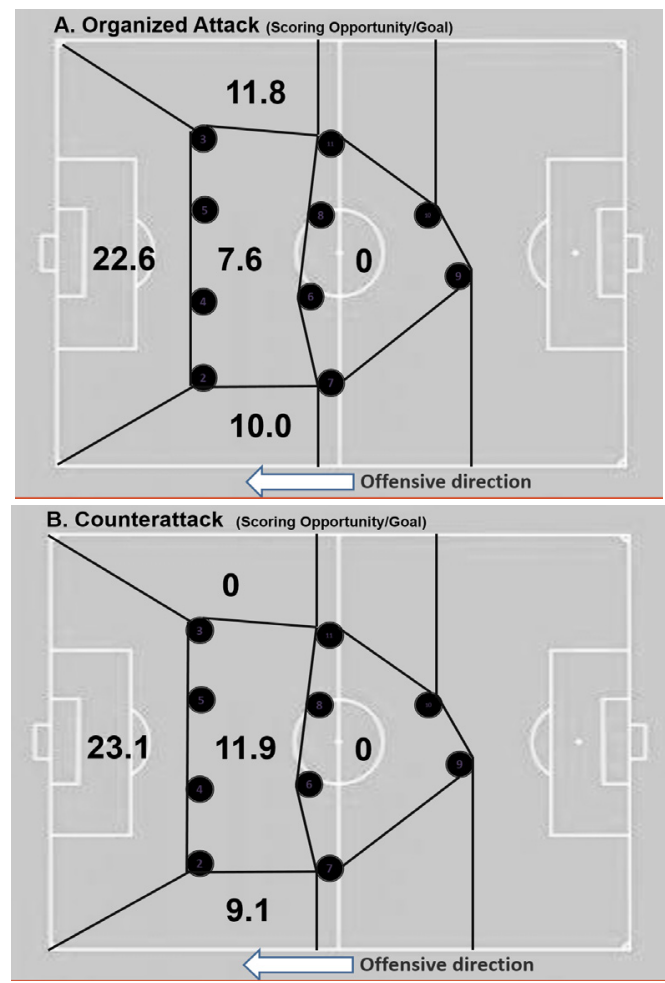

Figure 4. Ratio of goals scored for each invasive zone
Table 7. Scoring opportunities created by set pieces and goal scoring percentage

\begin{tabular}{lccc} 
& $n(\%)$ & \multicolumn{2}{c}{ Goal scoring } \\
Type of set pieces & & $n$ & $\%$ \\
& & & \\
Corner kick & $37(46.8)$ & 5 & 13.5 \\
Free kick & $36(45.6)$ & 6 & 16.7 \\
Penalty & $6(7.6)$ & 5 & 83.3
\end{tabular}

attacks (22.6\% and $23.1 \%)$, while the central defensive zone achieved $7.6 \%$ in organized attacks and $11.9 \%$ in counterattacks (Figure 4).

Among the set pieces that finished in scoring opportunity (Table 7 ), $46.8 \%$ were corner kicks, $45.6 \%$ were free kicks, and $7.6 \%$ were penalty kicks.

\section{DISCUSSION}

The aim of this study was to describe how scoring opportunities emerge and finish in United States MLS, taking into account not only offensive tactical indicators but also some defensive ones (such as initial pen- 
etration, initial opponent position, initial invasive zone, initial opponent number, initial pressure) of the opponent team.

Regarding the contextual characteristics of the sample, teams created more scoring opportunities by using organized attacks when they were losing than when drawing or winning. Also, when they were winning, the proportion of counterattacks was much higher than when drawing or winning. This fact agrees with previous research that showed how teams that were losing increased their possession, suggesting that they preferred to 'control' the game by dictating it [26]. Also, Lago-Ballesteros et al. [18] observed that when the team was drawing or winning, the probability of reaching the score-box decreased dramatically compared with the losing situation. These results may be bound with the fact that when teams were ahead, they decreased their possession, suggesting that they preferred to play counterattack.

It was found that $52.5 \%$ of scoring opportunities were created from open play possessions. This percentage is lower than the $65 \%$ of scoring opportunities started by means of transition play in the English Premier League [8] and the 58.5\% in the World Cup 2010 [10]. For restarts, set pieces created $21.9 \%$ of the total scoring opportunities, which remains in line with the 20.0\% found in the World Cup 2010 [10]. Previous research has suggested that set plays account for approximately $1 / 3$ of all goals scored [2, 3, 27], although the methodology of these studies did not take into account the differences between restarts and set pieces considered in the present study. On the other hand, this study demonstrates that organized attacks created a bigger proportion of scoring opportunities than counterattacks (25.3\%) and set pieces. In turn, recent studies observed how organized attack created more scoring opportunities during World Cup 2010 [10], as well as for Real Madrid CF and FC Barcelona during the 2012-2013 season [9]. Also, organized attack was the way that achieved more goals in 2006 World Cup [3] and 2004 [2] and 2012 Euro [27]. The fact that this type of attack has a higher proportion of sequences during the game may explain the higher proportion of goal scoring opportunities so that some studies observed that counterattacks occurred less frequently than organized attacks but were more effective to create offensive success [14, 18]. Castellano [28] and Castellano et al. [29] also showed that the number of game sequences composed by one single possession tended to increase in several national team tournaments from 1991 to 2006.

As for the initial tactical indicators that led to scor- ing opportunities, organized attacks were characterized by starting in pre-offensive zones on the field but not in invasive zones, taking into account the lines of the opponent. This fact shows the importance of analysing the opponent defensive positioning to know the real spatial context in which the tactical actions take place. The first action was mainly non-penetrative and the passing sequences to create scoring opportunity were predominantly long $(71.1 \%$ needed 4 or more passes and $42.6 \%$ needed 7 or more). On the other hand, counterattacks were also started in preoffensive zones on the field but in invasive zones over the opponent lines, performing initial penetrative actions and making very few passes $(70.3 \%$ had 3 or less passes). Similar results regarding tactical indicators used in counterattacks and organized attacks were found in the creation of scoring opportunities during the 2010 World Cup [10]. According to our results, successfully organized attacks occurred when the opponent was organized and the offensive behaviour was to be patient and not to penetrate immediately. Otherwise, for counterattacks, the situation of the opponent could require to penetrate as fast as possible to make the most of the invasion already achieved. For this reason, to analyse the position and behaviour of the opponent at the beginning of the possession is key for the players to make quick and appropriate decisions about penetrating or not penetrating, which will change the development of that team possession. In this sense, professional coaches who were interviewed in the study by Sarmento et al. [30] highlighted the importance of the first actions after gaining the ball so the team could overcome a great number of opposing players and create goal attempts. This could allow a longer duration of the possession, crucial to avoid losing the ball in transitions [31]. Some differences in the results obtained in the transitions when comparing with other studies [32, 33] could be explained by methodological differences (type of competition studied, observation instrument, and analysis).

In the finishing situation, penetrating passes from inside areas were the most frequent actions performed by the penultimate player in possession for both types of attack while the $28.1 \%$ of the total scoring opportunities were created after a cross. Other studies performed in national teams proved that more goals were scored after passing the ball from central areas than from wide areas [4, 34]. However, it is interesting to observe how our study found that wide areas and crosses were more frequent in organized attacks (32.1\%) than counterattacks (19.5\%), while the proportion of passes behind the defence 
was higher in counterattacks $(12.8 \%$ vs. $28.7 \%$, respectively). González-Ródenas et al. [10] also observed that wide areas were more used in organized attacks than counterattacks during the action prior to the creation of scoring opportunities. This study found a higher proportion of previous actions performed in wide areas to create scoring opportunities (46.5\%) in comparison with World Cup 2010 (35.0\%). The mentioned tactical differences may be a tactical characteristic of MLS or may be due to the different tactical scenarios provoked by the opposing team related or not with the fact of being a national team tournament or regular club league. For organized attacks, the defensive team would be more organized and compact, so the inside space would be more protected and it would make more sense to bring the ball to wide areas to progress and cross. Contrarily, the opponent would be more disorganized in a counterattack situation, so there would be more space to exploit in central areas.

For the final action, the central defensive zone (space between the defensive line and the midfielder line) was the most used to finish the attack but counterattacks achieved a deeper penetration over the opponent and had a greater proportion of scoring opportunities closer to the goal in comparison with organized attacks. This fact means that counterattacks can create clearer opportunities and this may explain the higher effectiveness achieved by this type of attack with respect to organized attacks in recent studies $[14,18]$. These results coincide with the study of Seabra and Dantas [22] in World Cup 2002 and González-Ródenas et al. [10] in World Cup 2010, who observed the central defensive zone as the most used space to assist and finish scoring chances. However, the present study has not found significant differences in the ratio of scoring opportunities and goals between the two types of attack. For both of them, the central back zone produced a higher ratio of goals (approximately $1 / 4$ ), followed by the central defensive zone (approximately 1/10). In this sense, previous research has found that a close distance to the goal, a great angle between the ball and the goal, and no pressure on the ball are key factors to assure a high ratio of goal scoring [19]. This fact is not surprising as the central back zone is the area where only the goalkeeper is between the ball and the goal and there are no defenders putting pressure on the player with the ball. This result highlights the importance of breaking the defensive line to increase the possibilities to score goals, so the ability of players to make runs in behind the defence, as well as the ca- pacity of making passes that are able to connect to these players are crucial for clearer scoring opportunities.

Regarding set pieces, corner kicks and free kicks produced $46.8 \%$ and $45.6 \%$ of the scoring opportunities created by set plays and $10.2 \%$ and $10.5 \%$ of the total scoring opportunities, respectively. This percentage of scoring opportunities is considerable if one takes into account that set pieces have a low frequency of occurrence during games as compared with organized attacks and counterattacks. Several studies have observed that ca. $20-30 \%$ of the total set pieces achieved a finish as a shot, although the effectiveness in terms of goal scoring has been reported to be around 1.5 and 3\% [35, 36]. Despite their low effectiveness, goals resulting from set pieces had a decisive impact on the final outcome of matches [35, 36]. Casal et al. reported that goals scored from corner kicks led to a draw or victory in $75 \%$ of cases [36]. We think that some features of set pieces could contribute to their important capacity to make scoring opportunities: the proximity to the goal, the offensive participation of defensive players with ability to head the ball, the directness to put the ball in the opposing penalty box, as well as the possibility to choose the right moment to initiate the play can explain the great impact that this type of attack can have during games in elite soccer.

Future investigations focused on other domestic leagues (e.g. La Liga, Premier League) would put more light into tactical features of the best club teams of the world and also would allow to compare different leagues.

Lastly, as limitations of the study, this paper is focused on notational data based on frequencies and percentages of actions occurred during the game that may not provide the whole understanding of the complex tactical behaviours taking place during a soccer game. Also, the study has practical applications for soccer coaches, who should take into account the position of the opponent when they design tactical drills to improve the different types of attack in soccer. To reproduce organized attacks, the opponent should have a compact defence, in low or medium block in order to make it difficult to penetrate in the first actions of the possession. On the other hand, training games that try to reproduce counterattacks should focus on creating tactical situations where the play starts in invasive zones of the opponent, making it possible to penetrate in the first actions and to finish in central areas of the field after progressing with 3 or less passes. 


\section{CONCLUSIONS}

The study shows how in random MLS matches, organized attacks created a greater proportion of scoring opportunities than counterattacks and set pieces. The scoring opportunities provided by organized attacks, in comparison with counterattacks: (a) started more frequently in non-invasive zones; (b) led to a greater proportion of non-penetrative actions; (c) carried out longer passing sequences; (d) used more the wide areas and performed more crosses prior to creating scoring opportunities; (e) achieved less penetration over the opponent when finishing the possession.

\section{Acknowledgments}

We acknowledge the support of a Spanish government project (Ministerio de Economía y Competitividad): La actividad física y el deporte como potenciadores del estilo de vida saludable: Evaluación del comportamiento deportivo desde metodologías no intrusivas [Grant number DEP2015-66069-P].

\section{Disclosure statement}

No author has any financial interest or received any financial benefit from this research.

\section{Conflict of interest}

Authors state no conflict of interest.

\section{References}

1. O’Donoghue PG. The use of feedback videos in sport. In: Dancs H, Hughes M, O’Donoghue PG (eds.), Performance analysis of sport 7. Cardiff: UWIC; 2006; 126137.

2. Yiannakos A, Armatas V. Evaluation of the goal scoring patterns in the European Championship in Portugal 2004. Int J Perform Anal Sport. 2006;6(1):178-188; doi: 10.1080/24748668.2006.11868366.

3. Armatas V, Yiannakos A. Analysis and evaluation of goals scored in 2006 World Cup. J Sport Health Res. 2010;2(2):119-128.

4. Smith RA, Lyons K. A strategic analysis of goals scored in open play in four FIFA World Cup football championships between 2002 and 2014. Int J Sports Sci Coach. 2017;12(3):398-403; doi:10.1177/1747954117710516.

5. James N, Mellalieu SD, Hollely C. Analysis of strategies in soccer as a function of European and domestic competition. Int J Perform Anal Sport. 2002;2(1):85103; doi: 10.1080/24748668.2002.11868263.

6. Tenga A, Ronglan LT, Bahr R. Measuring the effectiveness of offensive match-play in professional soccer. Eur J Sport Sci. 2010;10(4):269-277; doi: 10.1080/17461390 903515170 .
7. Hughes M, Franks I. Analysis of passing sequences, shots and goals in soccer. J Sports Sci. 2005;23(5):509514; doi: 10.1080/02640410410001716779.

8. Wright C, Atkins S, Polman R, Jones B, Lee S. Factors associated with goals and goal scoring opportunities in professional soccer. Int J Perform Anal Sport. 2011;11(3): 438-449; doi: 10.1080/24748668.2011.11868563.

9. López Bondia I, González Rodenas J, Calabuig Moreno F, Pérez-Turpin JA, Aranda Malavés R. Creating goal scoring opportunities in elite soccer. Tactical differences between Real Madrid CF and FC Barcelona. Retos. 2017;32:233-237. Available from: https://recyt.fecyt.es/index.php/retos/article/view/56467.

10. González-Ródenas J, López Bondia I, Calabuig Moreno F, Aranda Malavés R. Tactical indicators associated with the creation of scoring opportunities in professional soccer [in Spanish]. Cult Cienc Deporte. 2015;10(30): 215-225; doi: 10.12800/ccd.v10i30.590.

11. González-Ródenas J, López-Bondia I, Calabuig F, PérezTurpin JA, Aranda R. Association between playing tactics and creating scoring opportunities in counterattacks from United States Major League Soccer games. Int J Perform Anal Sport. 2016;16(2):737-752; doi: 10.1080/24748668.2016.11868920.

12. Mackenzie R, Cushion C. Performance analysis in football: a critical review and implications for future research. J Sports Sci. 2013;31(6):639-676; doi: 10.1080/ 02640414.2012 .746720$.

13. Olsen E, Larsen O. Use of match analysis by coaches. In: Reilly T, Hughes M, Bangsbo J (eds.), Science and football III. London: E. and F.N. Spon Ltd.; 1997; 209-222.

14. Tenga A, Holme I, Ronglan LT, Bahr R. Effect of playing tactics on achieving score-box possessions in a random series of team possessions from Norwegian professional soccer matches. J Sports Sci. 2010;28(3):245-255; doi: 10.1080/02640410903502766.

15. Strutner M, Parrish C, Nauright J. Making soccer 'Major League' in the USA and beyond: Major League Soccer's first decade. Sport Hist Rev. 2014;45(1):23-36; doi: 10.1123/shr.2012-0017.

16. Urbaniak GC, Plous S. Research Randomizer (Version 4.0) [Computer software]. Available from: http://www. randomizer.org/.

17. Pollard R, Reep C. Measuring the effectiveness of playing strategies at soccer. The Statistician. 1997;46(4): 541-550; doi: 10.1111/1467-9884.00108.

18. Lago-Ballesteros J, Lago-Peñas C, Rey E. The effect of playing tactics and situational variables on achieving score-box possessions in a professional soccer team. $\mathrm{J}$ SportsSci.2012;30(14):1455-1461;doi:10.1080/02640414. 2012.712715.

19. Pollard R, Ensum J, Taylor S. Estimating the probability of a shot resulting in a goal: the effects of distance, angle and space. Int J Soccer Sci. 2004;2(1):50-55.

20. Gómez MA, Gómez-Lopez M, Lago C, Sampaio J. Effects of game location and final outcome on game-related statistics in each zone of the pitch in profession- 


\section{HUMAN MOVEMENT}

J. Gonzalez-Rodenas et al., Scoring attempts in US Major League Soccer

al football. Eur J Sport Sci. 2012;12(5):393-398; doi: 10.1080/17461391.2011.566373.

21. Barreira D, Garganta J, Guimarães P, Machado J, Anguera MT. Ball recovery patterns as a performance indicator in elite soccer. J Sports Eng Tech. 2014;228(1): 61-72; doi: 10.1177/1754337113493083.

22. Seabra F, Dantas L. Space definition for match analysis in soccer. Int J Perform Anal Sport. 2006;6(2):97113; doi: 10.1080/24748668.2006.11868376.

23. González-Rodenas J. Analysis of the tactical performance in the offensive collective play in the World Cup 2010 [in Spanish]. Unpublished doctoral dissertation. Valencia: University of Valencia; 2013.

24. Gabin B, Camerino O, Anguera MT, Castañer M. Lince: multiplatform sport analysis software. Procedia Soc Behav Sci. 2012;46:4692-4694; doi: 10.1016/j.sbspro. 2012.06.320.

25. Altman DG. Some common problems in medical research. In: Altman DG (ed.), Practical statistics for medical research. London: Chapman \& Hall; 1991; 403-409.

26. Lago C, Martin R. Determinants of possession of the ball in soccer. J Sports Sci. 2007;25(9):969-974; doi: 10.1080/02640410600944626.

27. Mitrotasios M, Armatas V. Analysis of goal scoring patterns in the 2012 European Football Championship. Sport J. 2014;1:1-11.

28. Castellano J. Analysis of football possessions in soccer: frequency, duration and transition [in Spanish]. Motricidad. Eur J Hum Mov. 2008;21:189-207.

29. Castellano J, Perea A, Alvarez D. Transitions in the possession of the ball in football: from the possible to the probable [in Spanish]. Apunts Educ Fisica Deportes. 2009;95(1):75-81.

30. Sarmento H, Anguera MT, Pereira A, Marques A, Campanico J, Leitão J. Patterns of play in the counterattack of elite football teams - a mixed method approach. Int J Perform Anal Sport. 2014;14(2):411-427; doi: 10.1080/ 24748668.2014.11868731.

31. Casal CA, Andujar MA, Losada JL, Ardá T, Maneiro R. Identification of defensive performance factors in the 2010 FIFA World Cup South Africa. Sports. 2016;4(4): 54; doi: 10.3390/sports4040054.

32. Casal CA. How to improve offensive efficiency in football: offensive transitions [in Spanish]. Berlin: EAE; 2011.

33. Andujar MA. The defensive transition in elite football [in Spanish]. Berlin: EAE; 2016.

34. Horn R, Williams AM, Grant A. Analysis of France in World Cup 1998 and Euro 2000. Insight. 2000;4(1): 40-43.

35. Casal AC, Maneiro R, Ardá T, Losada JL, Rial A. Effectiveness of indirect free kicks in elite soccer. Int J Perform Anal Sport. 2014;14(3):744-760; doi: 10.1080/ 24748668.2014.11868755.

36. Casal CA, Maneiro R, Ardá T, Losada JL, Rial A. Analysis of corner kick success in elite football. Int J Perform AnalSport.2015;15(2):430-451; doi:10.1080/24748668. 2015.11868805. 